\title{
Up-Down Quark Mass Difference Effect in Nuclear Many-Body Systems
}

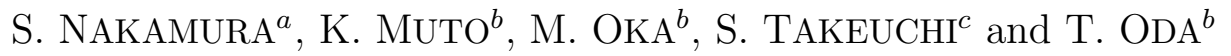 \\ a Institute for Nuclear Study, University of Tokyo, Tokyo 188, Japan \\ ${ }^{b}$ Department of Physics, Tokyo Institute of Technology, Tokyo 152, Japan \\ ${ }^{c}$ Department of Public Health and Environmental Science, \\ Tokyo Medical and Dental University, Tokyo 113, Japan
}

\begin{abstract}
A charge-symmetry-breaking nucleon-nucleon force due to the up-down quark mass difference is evaluated in the quark cluster model. It is applied to the shell-model calculation for the isovector mass shifts of isospin multiplets and the isospin-mixing matrix elements in 1s0d-shell nuclei. We find that the contribution of the quark mass difference effect is large and agrees with experiment. This contribution may explain the Okamoto-Nolen-Schiffer anomaly, alternatively to the meson-mixing contribution, which is recently predicted to be reduced by the large off-shell correction.
\end{abstract}

PACS numbers: 21.10.Hw, 24.85.+p, 21.60.Cs 
Charge-symmetry-breaking (CSB) terms is required in the nuclear force for explaining several phenomena, e.g., the difference between the proton-proton and neutron-neutron scattering lengths [1] and the anomaly of mass differences of several mirror nuclei, called Okamoto-Nolen-Schiffer (ONS) anomaly [2, 3, 田, 可. These experimental data imply that the nuclear force between two neutrons is slightly more attractive than between two protons.

Three of the present authors have made an extensive analysis of isovector mass shifts and isospin-mixing matrix elements in 1s0d-shell nuclei. It was shown that experimental values of these quantities are well explained [6] by a short-range CSB force, but not by a long-range force. According to these findings, we look for the origin of a short-range CSB force in this report.

The experimental isovector mass shift, $b(\nu, T)$, is calculated by letting the isospin multiplet mass equation,

$$
E\left(\nu, T, T_{z}\right)=a(\nu, T)+b(\nu, T) T_{z}+c(\nu, T) T_{z}^{2},
$$

reproduce the masses, $E\left(\nu, T, T_{z}\right)$, of the $2 T+1$ members of the nuclear isospin multiplet. In the above equation, $\nu$ represents the quantum numbers other than the isospin, $T$, and its third component, $T_{z}$.

Several well-known CSB sources have contributions to the isovector mass shifts. Before considering the nuclear-CSB force, we subtract the contributions of the electromagnetic interactions (EM) and of the isovector single particle energy (ISPE). They are calculated by the analysis of 1s0d-space shell model [6, 7]. The EM contribution is evaluated in taking account of the Coulomb force between the protons with the charge form factor correction, the electromagnetic spin-orbit force and the magnetic spin-spin contact interaction with the magnetic form factor of the nucleon. The explicit formulae are given in ref. [6]. The ISPE represents the CSB interactions between the valence nucleon and the ${ }^{16} \mathrm{O}$-core. Subtracting them, we obtain the "experimental" data, shown in Fig. 1, which is to be compared with the nuclear-CSB contribution.

We find a systematic behaviour, called "zigzag behaviour", i.e., that the "experimental" values of the isodoublets are reduced for $A=4 n+1$ while for $A=4 n+3$ they are enhanced. It is demonstrated in the previous studies [6, 7] that a short-range CSB force is the most probable source of the "zigzag behaviour".

A short-range nuclear-CSB force can be provided by the exchange of the mixed $\rho-\omega$ complex. It is extremely short-ranged, and explains at least half of the "zigzag behaviour" [7]. The meson-mixing potential is also used to explain other phenomena, e.g., the ONS 
anomaly [8, 9, 10]. Goldman et al. [1] argued, however, that an off-shell correction reduces the meson-mixing amplitude by a large factor. The correction is so large as to kill the mesonmixing contribution. Other calculations [12, 13, 14 also verify such an off-shell effect. It is, however, still controversial, because another analysis indicates the strong off-shell effect being inconsistent with the observed $q^{2}$-dependence of $\rho-\gamma^{*}$ coupling [15]. Further studies seem to be necessary.

In the present article, we assume that the meson-mixing contribution is negligible, and examine another short-range CSB force due to the up-down quark mass difference, called quark effect (QE). Precisely speaking, it is a contribution of the quark mass difference in the gluon-exchange interaction between the valence quarks, while the meson-mixing may contain effects of the quark mass difference as well [1]. Such a quark CSB force has been used to explain the difference between the proton-proton and neutron-neutron scattering lengths [16] and the ONS anomaly [17]. In this study, we apply the quark CSB force to the shell model calculation for the mass shifts of nuclear isospin multiplets.

The quark CSB potential is calculated in the nonrelativistic potential quark model of baryons [16]. The model consists of the standard interaction hamiltonian, which contains a quark confining potential as well as one-gluon exchange interaction. The confining potential is assumed to be independent of isospin, i.e., flavour of the quarks [1, 16]. The one-gluon exchange interaction contains an isospin-symmetry-breaking term in the hyperfine contact interaction,

$$
H_{\mathrm{HC}}^{\left(\mathrm{q}_{i} \mathrm{q}_{j}\right)}=-\left(\lambda_{i} \cdot \lambda_{j}\right) \frac{\pi \alpha_{\mathrm{s}}}{6 m_{i} m_{j}}\left(\vec{\sigma}_{i} \cdot \vec{\sigma}_{j}\right) \delta^{(3)}\left(r_{i j}\right),
$$

where $\lambda_{i}, \vec{\sigma}_{i}$ and $m_{i}$ are the colour $\mathrm{SU}(3)$ generator, Pauli spin matrix and mass of the constituent quark, $\mathrm{q}_{i}$, and $\alpha_{\mathrm{s}}$ is the strong coupling constant. Other terms are estimated to have negligible contributions to the isospin-dependent nucleon-nucleon (NN) interaction [16]. This contact interaction yields a short-range NN force which has the range of the nucleon size.

The two nucleon system is represented by a quark-cluster wave function composed by two three-quark clusters [16]. The internal wave function of each nucleon is approximated by a Gaussian, and the internal variables are integrated out to obtain the NN potential from the quark-quark interaction. The obtained potential depends on the NN relative coordinate as well as the spin and isospin quantum numbers.

It is found in the calculation of Chemtob and Yang (see Fig. 2 of ref. [16]) that the local 
term of the hyperfine contact interaction is the leading term and represents the whole CSB interaction approximately. In this article, therefore, we deal only with the local term of the hyperfine contact interaction, and the other terms are neglected.

In order to apply the potential to the shell-model calculation, we have to prepare the potentials for higher partial waves than the S-states. They are numerically calculated [18], and we obtain a quark CSB potential,

$$
v_{\mathrm{QE}}^{\left(\mathrm{N}_{1} \mathrm{~N}_{2}\right)}=\sqrt{\frac{3}{\pi}} \frac{\beta^{3} \alpha_{\mathrm{s}}}{\hat{m}^{2}} \exp \left(-\frac{3}{4} \beta^{2} r_{12}^{2}\right) \frac{1}{4}\left[\tau_{z}(1)+\tau_{z}(2)\right] \frac{\delta m}{\hat{m}}\left[1-\frac{5}{27} \vec{\sigma}(1) \cdot \vec{\sigma}(2)\right],
$$

where $\beta^{-1}$ is the nucleon size parameter of the three-quark cluster, and $r_{12}$ is the distance between the centers of the two clusters, $\tau_{z}(i)$ is the third component of the Pauli matrix for the nucleon isospin, and $\vec{\sigma}(i)$ is for the nucleon spin. $\delta m=m_{\mathrm{d}}-m_{\mathrm{u}}$ is the up-down quark mass difference and $\hat{m}=\frac{1}{2}\left(m_{\mathrm{d}}+m_{\mathrm{u}}\right)$ is the average of the masses. The input parameters are taken from ref. [16], $\delta m=6 \mathrm{MeV}, \hat{m}=330 \mathrm{MeV}, \alpha_{\mathrm{s}}=1.624$ and $\beta^{-1}=0.616 \mathrm{fm}$.

The nuclear matrix element of the QE potential gives the isovector mass shift,

$$
b^{\mathrm{QE}}(\nu, T)=\frac{1}{\sqrt{(2 T+1) T(T+1)}}\left\langle\nu, T\left\|v_{\mathrm{QE}}\right\| \nu, T\right\rangle,
$$

in the first order perturbation, where the matrix element $\left\langle\nu, T \| v_{\mathrm{QE}}|| \nu, T\right\rangle$ is reduced with respect to the isospin.

The nuclear wave function, $|\nu, T\rangle$, is calculated [6, 7] with Wildenthal's effective hamiltonian [19] in the complete 1s0d-shell space. Because the short-range QE potential is integrated, the calculation is sensitive to the short-range structure of the relative wave function of the two nucleons. We include a short-range correlation by a correlation function of ref. 20] multiplied to the relative two-nucleon wave function.

The calculated QE contributions are shown in Fig. 2. The QE contributions are around $100 \mathrm{keV}$, and the average ratio of QE/Coulomb contributions for 143 multiplets is $5.3 \%$. This result is consistent with the expected contribution which is introduced phenomenologically to explain the ONS anomaly in literatures [4, 5], and consistent with the previous calculation of the quark effect for the anomaly [17].

The "zigzag behaviour" seen in the "experimental" mass shifts (Fig. 1) is also reproduced in the calculation (Fig. 2). Namely, the quark contributions are larger for the $A=4 n+3$ isodoublets, and smaller for $A=4 n+1$. This behaviour is due to the short-range nature of the quark CSB force [6]. 
We need to comment on the calculation of the ISPE contribution. The ISPE used in Fig. 1 is determined by a $\chi^{2}$-fitting to the experimental mass shifts, including the QE contribution by the prescription of ref. [7]. By this procedure, the ISPE could be adjusted to produce the "zigzag behaviour" given by the QE contribution, even if the original data would not have a "zigzag" feature. To examine this, we have made another $\chi^{2}$-fitting without any nuclear-CSB contribution, and still found the "experimental zigzag behaviour" (see ref.[6, []).

The quark CSB potential of eq.(2) is also applied to off-diagonal matrix elements, i.e., isospin-mixing matrix elements. The results are given in Table 1, with experimental values extracted from the strength of the isospin-forbidden beta decays. We find that the calculated matrix elements agree with most of the experimental values.

To the off-diagonal matrix elements, the quark contribution is found to be large, comparable with the electromagnetic contributions (EM) in the table, which are roughly equal to the contributions of the Coulomb force. By contrast, the mass shifts are dominated by the Coulomb contributions. Such large contributions to the off-diagonal matrix elements can be attributed to the short-range feature of the quark CSB force [21, 6].

In particular, the beta decay of ${ }^{24} \mathrm{Al}$ (Table 1) indicates a large isospin-mixing in an excited state of the daughter nucleus ${ }^{24} \mathrm{Mg}$. Our calculation also gives a large QE contribution to this matrix element, and thus is consistent with experiment.

In summary, the isovector mass shifts and isospin-mixing matrix elements relevant to the isospin-forbidden beta decays are calculated considering the effect of the up-down quark mass difference in the direct gluon-exchange process. The charge-symmetry-breaking (CSB) nucleon-nucleon potential due to the quark mass difference is constructed with the quark cluster model, and is applied to the shell model calculation of the 1s0d-shell space.

The quark contributions are found to be about $5 \%$ of the Coulomb contributions to the isovector mass shifts of the nuclear isospin multiplets. It is consistent with the expected contributions [4, 5] to explain the anomaly of mass differences of the mirror nuclei, known as the Okamoto-Nolen-Schiffer anomaly. Also, the quark contribution is found to have a systematic behaviour in the contributions to the mass shifts of the isospin doublets, i.e., for $A=4 n+1$ the mass shifts are reduced while for $A=4 n+3$ they are enhanced. This behaviour is consistent with the experimental one.

The calculated values of the isospin-mixing matrix elements are consistent with the most 
of the experimental values extracted from the isospin-forbidden beta decays. The quark CSB force is found to have large contributions to the isospin-mixing matrix elements, comparable with the Coulomb contributions. A particularly large value of the experimental matrix element of ${ }^{24} \mathrm{Mg}$ is found to be explained by the large quark contribution. These findings strongly suggest the existence of a short-range CSB interaction.

The quark mass difference effect is, of course, not a unique possible source of the shortrange CSB force. However, it seems to have large contribution to the observables, and have a favourable feature for explaining the known data.

The numerical calculations were performed with the FACOM M780 computer system at the Institute for Nuclear Study, University of Tokyo. This study is partly supported by a Grant-in-Aid for Scientific Research (05243204 and 06234206) from the Ministry of Education, Science and Culture (Monbusho), and one of the authors (S.N.) is supported by a scholarship from the Soryushi Shogakukai.

\section{References}

[1] G.A.Miller, B.M.K.Nefkens and I.Šlaus, Phys. Rep. 194, 1 (1990)

[2] K.Okamoto, Phys. Lett. 11, 150 (1964)

[3] J.A.Nolen,Jr. and J.P.Schiffer, Ann. Rev. Nucl. Sci. 19, 471 (1969)

[4] H.Sato, Nucl. Phys. A269, 378 (1976)

[5] S.Shlomo, Rep. Prog. Phys. 41, 957 (1978)

[6] S.Nakamura, K.Muto and T.Oda, Nucl. Phys. A575, 1 (1994)

[7] S.Nakamura, Thesis, Tokyo Institute of Technology (1994)

[8] P.G.Blunden and M.J.Iqbal, Phys. Lett. B198, 14 (1987)

[9] Y.Wu, S.Ishikawa and T.Sasakawa, Phys. Rev. Lett. 64, 1875 (1990)

[10] T.Suzuki, H.Sagawa and A.Arima, Nucl. Phys. A536, 141 (1992)

[11] T.Goldman, J.A.Henderson and A.W.Thomas, Few-Body Systems 12, 123 (1992) 
[12] J.Piekarewicz and A.G.Williams, Phys. Rev. C47, R2462 (1993)

[13] G.Krein, A.W.Thomas and A.G.Williams, Phys. Lett. B317, 293 (1993)

[14] T.Hatsuda, E.M.Henley, Th.Meissner and G.Krein, Phys. Rev. C49, 452 (1994)

[15] G.A.Miller, U. Washington preprint, DOE/ER/40427-09-N94

[16] M.Chemtob and S.N.Yang, Nucl. Phys. A420, 461 (1984)

[17] V.Koch and G.A.Miller, Phys. Rev. C31, 602 (1985); C32, 1106 (1985)

[18] S.Takeuchi, K.Shimizu and K.Yazaki, Nucl. Phys. A504, 777 (1989)

[19] B.H.Wildenthal, Prog. Part. Nucl. Phys., vol.11, ed. D.H.Wilkinson (Pergamon, Oxford, 1984) p.5

[20] G.A.Miller and J.E.Spencer, Ann. Phys. 100, 562 (1976)

[21] S.Nakamura, K.Muto and T.Oda, Phys. Lett. B311, 15 (1993)

[22] A.H.Wapstra and G.Audi, Nucl. Phys. A432, 1 (1985);

A.H.Wapstra, G.Audi and R.Hoekstra, At. Data Nucl. Data Tables 39, 281 (1988)

[23] F.Ajzenberg-Selove, Nucl. Phys. A475, 1 (1987)

[24] P.M.Endt and C.van der Leun, Nucl. Phys. A310, 1 (1978);

P.M.Endt, Nucl. Phys. A521, 1 (1990)

[25] K.Muto, S.Nakamura and T.Oda, Prog. Theor. Phys. 91, 287 (1994)

[26] D.M.Perlman, L.Grodzins and C.E.Thorn, Phys. Rev. C18, 2333 (1978)

[27] S.Raman, T.A.Walkiewicz and H.Behrens, At. Data Nucl. Data Tables 16, 451 (1975)

[28] A.Ray, C.D.Hoyle and E.G.Adelberger, Nucl. Phys. A378, 29 (1982)

[29] C.D.Hoyle, E.G.Adelberger, J.S.Blair, K.A.Snover, H.E.Swanson and R.D.von Lintig, Phys. Rev. C27, 1244 (1983)

[30] D.E.Alburger and E.K.Warburton, Phys. Rev. C20, 793 (1979) 
Table 1 Isospin-mixing matrix elements in $\mathrm{keV}$ and their decompositions into the contributions of quark effect (QE), electromagnetic interactions (EM) and others. The EM consists of a large Coulomb contribution plus the other small contributions, and contains the isotensor terms of the electromagnetic interactions. "Other" consists of the ISPE contribution and the isotensor contribution due to the pion mass difference [25]. Experimental values are extracted from the strengths of isospin-forbidden beta decays of the given initial states [26]-30].

\begin{tabular}{lrrrrrrr}
\hline initial state & ${ }^{19} \mathrm{O}$ & ${ }^{20} \mathrm{~F}$ & ${ }^{24} \mathrm{Al}^{\mathrm{m}}$ & ${ }^{24} \mathrm{Na}$ & ${ }^{24} \mathrm{Al}$ & ${ }^{27} \mathrm{Mg}$ & ${ }^{28} \mathrm{Mg}$ \\
\hline $\mathrm{QE}$ & -12.2 & 11.5 & 7.4 & 5.5 & 36.8 & 2.6 & -8.2 \\
EM & 3.3 & 8.3 & 10.0 & 6.2 & 43.3 & -16.0 & -19.2 \\
other & 25.5 & -11.6 & -8.3 & -3.3 & -21.6 & 41.1 & 67.2 \\
total & 16.6 & 8.2 & 9.1 & 8.5 & 58.5 & 27.8 & 39.7 \\
experiment & $20(10)$ & $14_{-14}^{+29}$ & $49(5)$ & $5.4(22)$ & $106(40)$ & $3.6_{-3.6}^{+57}$ & $20.6(16)$ \\
\hline
\end{tabular}

\section{Figure captions:}

Fig. 1 The experimental isovector mass shifts [22, 23, 24] after subtracting the electromagnetic contributions and the isovector single-particle-energy contribution in keV. The isodoublets $(T=1 / 2)$ are denoted by the filled circles, and the others $(T>1 / 2)$ are by the crosses.

Fig. 2 The quark contribution to the isovector mass shifts in keV. Notations are the same as in Fig. 1. 


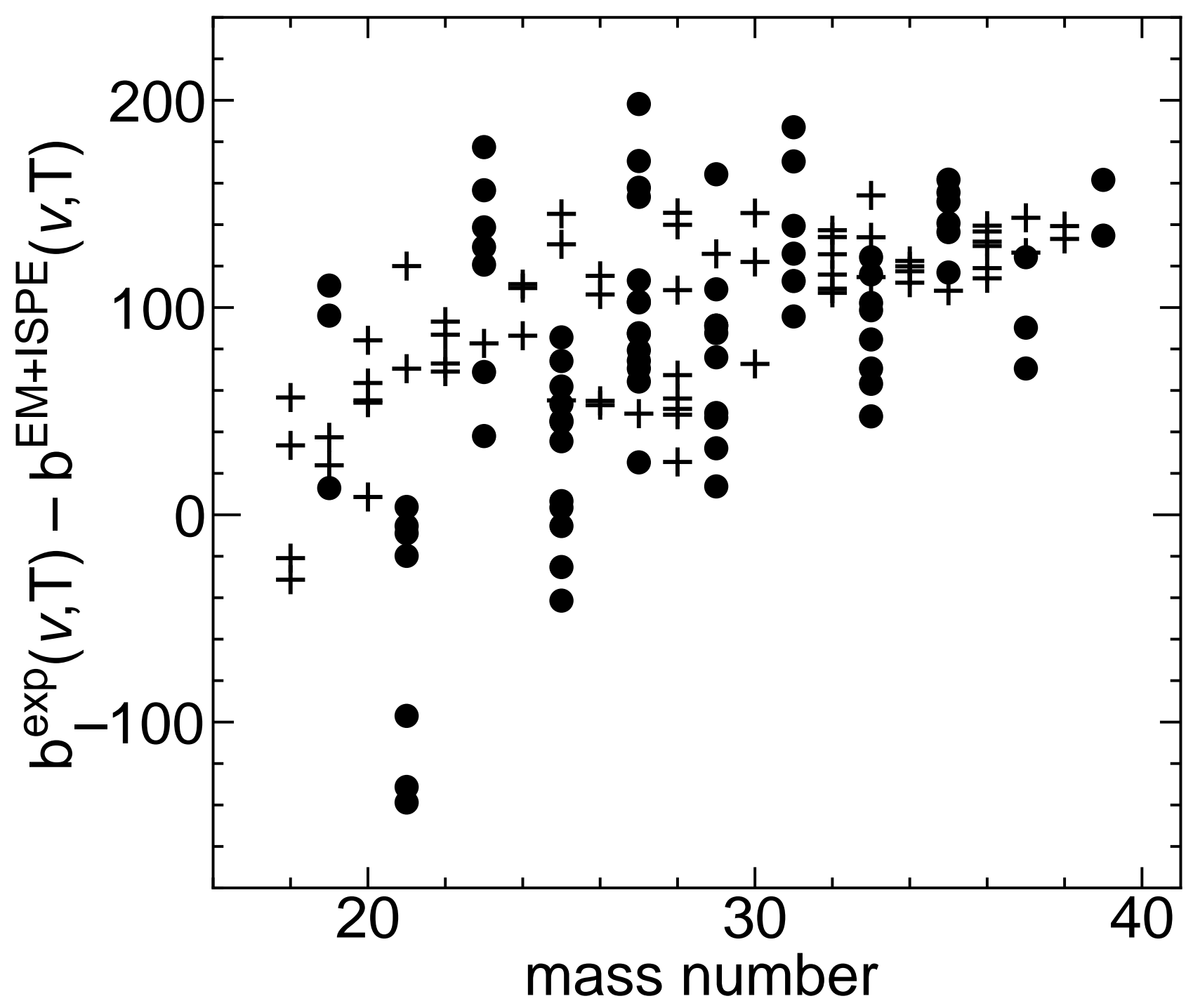




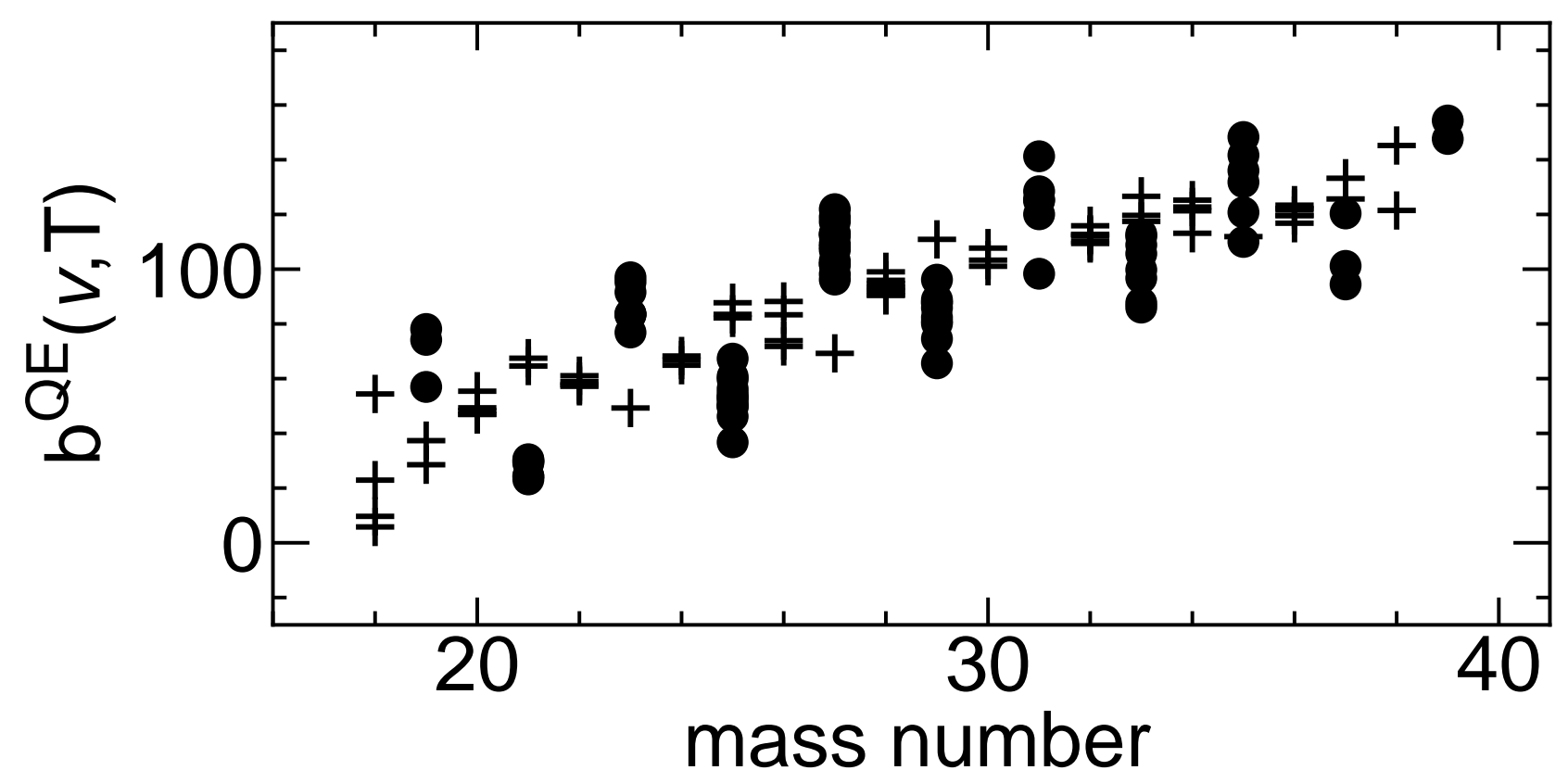


This figure "fig1-1.png" is available in "png" format from: http://arxiv.org/ps/nucl-th/9502013v1 
This figure "fig1-2.png" is available in "png" format from: http://arxiv.org/ps/nucl-th/9502013v1 